\title{
Head-On Collision of Neutron Stars As A Thought Experiment
}

\author{
Stuart L. Shapiro \\ Departments of Physics and Astronomy, 8 NCSA, University of Illinois at Urbana-Champaign, Urbana, IL 61801
}

\begin{abstract}
The head-on collision of identical neutron stars from rest at infinity requires a numerical simulation in full general relativity for a complete solution. Undaunted, we provide a relativistic, analytic argument to suggest that during the collision, sufficient thermal pressure is always generated to support the hot remnant in quasi-static stable equilibrium against collapse prior to slow cooling via neutrino emission. Our conclusion is independent of the total mass of the progenitors and holds even if the remnant greatly exceeds the maximum mass of a cold neutron star.
\end{abstract}

\section{INTRODUCTION}

Binary neutron stars are among the most promising sources for gravitational wave laser interferometers now under construction, like LIGO, VIRGO, GEO and TAMA. This fact has motivated an intense theoretical effort to understand their dynamical evolution and predict the gravitational waveforms emitted during their inspiral and coalescence.

Here we focus on the final collision and coalescence of the two stars. The complex interplay between hydrodynamics and gravitation, even in the idealized adiabatic case, requires that this late epoch be tackled by numerical means. The numerical integrations must be carried out in full general relativity even for a qualitative, let alone a quantitative, understanding of the key phases of the final evolution. Strong-field phenomena characterizing the final stages of binary neutron star evolution, like gravitational collapse leading to black hole formation, are not addressed at all by Newtonian theory.

Consider the following three strong-field questions: Do binary neutron stars collapse to black holes prior to contact? If not, does the remnant undergo gravitational collapse immediately following the merger whenever its rest mass exceeds the maximum equilibrium mass of an isolated cold, rotating neutron star, or is the collapse delayed? What is the final fate of a collapsing remnant if its angular momentum exceeds the Kerr limit, $J / M^{2} \leq 1$ ? None of these questions can be answered via a Newtonian simulation. The answers have important consequences for the design of advanced detectors and theoretical templates for measuring gravitational waves during the late epochs following collision, as well as other astrophysical consequences (e.g., gamma-ray burst models).

Here we address the second of the three questions posed above. To simplify the analysis we treat the simplest merger scenario - the head-on collision of two identical, nonspinning, cold neutron stars which fall together from rest at infinity. The stars may have a combined rest mass which greatly exceeds the hydrostatic equilibrium mass limit for an isolated, nonrotating, spherical star governed by the same cold equation of state. Even this problem requires a numerical simulation in full general relativity for a complete solution. However, by making a suitable set of simplifying approximations, we present an analytic argument to determine the fate of the remnant immediately following merger. Our calculation may provide a useful comparison for a $2 \mathrm{D}$ or $3 \mathrm{D}$ relativistic code which can perform such a simulation.

\section{ALTERNATIVE SCENARIOS}

Following the head-on collision of the two neutron stars, two recoil shocks form at the point of contact and propagate back into each star along the collision axis. Matter passing through the shock is heated and decelerated, as its kinetic energy is converted into thermal energy. The configuration is not in equilibrium following the passage of the initial recoil shocks, but undergoes oscillations, damped by acoustic (viscous) dissipation and gravitational radiation reaction forces. Only a small fraction of the total rest mass escapes from the system and an even smaller fraction of the total mass-energy is carried off by gravitational waves.

Two questions arise: Is the thermal pressure generated by shock and acoustic dissipation sufficient to support the merged remnant in hydrostatic equilibrium prior to cooling by neutrino dissipation? Does the answer depend on the total rest mass and its relation to the maximum rest mass of an isolated, equilibrium mass supported by the same initially cold equation of state?

Consider two alternative scenarios. In the first we suppose that the shock-generated thermal pressure is sufficiently large to prevent the remnant from undergoing immediate collapse, allowing it instead to settle down to quasi-equilibrium in a few dynamical timescales $(\sim \mathrm{msec})$ following the initial oscillations. By then, the bulk of the kinetic energy will have been converted into thermal energy, the rest having been emitted as gravitational waves. The star will relax to a hot, quasi-static, spherical equilibrium state. This equilibrium state will last many dynamical timescales because the thermal neutrinos, which eventually carry off the thermal energy, leak out slowly, on a neutrino diffusion timescale $(\sim 10 \mathrm{sec})$. As the star cools, it slowly contracts. If its total rest mass is less than the critical maximum value, it will evolve to a static, cold endpoint configuration and cease all further contraction. If its rest mass exceeds the critical value it must ultimately undergo catastrophic collapse to a Schwarzschild 
black hole. Since the quasi-static configuration is spherical and not too far from the Schwarzschild radius at the onset of this final 'delayed' collapse, the implosion will remain nearly spherical and generate little gravitational radiation.

Now consider the alternative scenario, for which the thermal pressure generated by shock heating is insufficient to support the star in quasi-static equilibrium. The star will then collapse promptly to a black hole on the relatively short dynamical timescale, and the bulk of the thermal energy and neutrinos will go down the hole. Unable to achieve dynamical equilibrium, the compressed remnant will never relax to a spherical shape so that its implosion will be nonspherical. During the implosion, the mass may then emit a significant final pulse of gravitational radiation before forming a static Schwarzschild black hole.

We show below that the first scenario is energetically feasible, i.e, the merged remnant can reside in a hot, quasi-static stable equilibrium phase lasting many dynamical timescales prior to cooling and contraction. Surprisingly, our conclusion is independent of the total rest mass of the object. Hence, even if the remnant mass greatly exceeds the maximum mass of a cold neutron star, the quasi-equilibrium will be stable and the collapse can be delayed considerably.

\section{KEY ASSUMPTIONS}

We assume that all of the original bulk kinetic energy at impact is dissipated into internal energy. Our goal is to assess whether the resulting pressure forces are capable of holding a nonrotating spherical remnant in stable equilibrium. We treat the collision and merger adiabatically and we assume that the combined rest mass and total mass-energy of the system is strictly conserved. We neglect any net outflow of baryons from the system, as well as the loss of energy via gravitational waves and neutrinos.

We adopt an adiabatic equation of state in the form

$$
P=K(s) \rho_{0}^{\Gamma},
$$

where $P$ is the pressure, $\rho_{0}$ is the rest-mass density, $\Gamma$ is the adiabatic index, assumed constant, and $K$ is a function of the specific entropy, $s$. We assume that the approaching stars are cold with $s=0$ and take $K(0)=K_{\text {cold }}$ to be a constant. We take the cold equation of state which supports the original neutron stars to be a polytrope, with polytropic index $n$ given by $\Gamma=1+1 / n$. We assume that the value of $\Gamma$ defining the initial polytropic equilibrium and the value associated with subsequent adiabatic changes are identical, as is assumed in most hydrodynamic numerical simulations of adiabatic gases. Adopting this assumption permits us to make a direct comparison with these simulations, as well as provide a simplified analytic treatment of the collision. The correct equation of state of hot, nuclear matter is still uncertain, and the actual variation of $\Gamma$ during compression and shock heating is not known. We note that $\Gamma$ remains strictly constant and equal to $5 / 3$ when ideal, degenerate, nonrelativistic neutrons are heated to nonrelativistic temperatures by the shock. Following the passage of all the shock and acoustic waves and the accompanying generation of entropy throughout the matter, we approximate the function $K(s)$ appearing in Eq. (11) by a new constant, $K(s)=K_{\text {hot }}$ 画. We thus treat the merged remnant as a new, hot spherical polytrope once it settles down. Below we determine $K_{\text {hot }}>K_{\text {cold }}$ uniquely in terms of $K_{\text {cold }}$ for any $n$.

Some of the above assumptions can be calibrated by comparing with existing Newtonian hydrodynamic simulations [1] [2] [3]. The results of Rasio and Shapiro (hereafter RS; [2]), who treated the adiabatic case $\Gamma=2$, are typical. They found that at most $5 \%$ of the total rest mass eventually escapes and that the remnant settles down to a hot, nearly spherical equilibrium state. The loss of energy via gravitational waves is about $\Delta E / M=2.4 \times 10^{-3} M_{1.4} R_{10}^{-7 / 2}$, where $M_{1.4}$ is the mass of one star in units of $1.4 M_{\odot}$ and $R_{10}$ is the radius in units of $10 \mathrm{~km}$. Hence, the assumptions of conserved rest-mass and total mass-energy during the initial dynamical epoch appear to be reasonable.

\section{NEWTONIAN ANALYSIS}

When two identical polytropes, each of (rest) mass $M_{\text {cold }}$, radius $R_{\text {cold }}$ and gas constant $K_{\text {cold }}$, collide headon from rest at infinity, the total initial energy of the system (excluding rest-mass energy) is given by

$$
E_{\text {cold }}=-\frac{(3 \Gamma-4)}{(5 \Gamma-6)} \frac{M_{\text {cold }}^{2}}{R_{\text {cold }}} \times 2 .
$$

Here and throughout we set $G=c=1$. The final remnant settles into a new polytropic configuration of the same index whose energy is given by

$$
E_{\mathrm{hot}}=-\frac{(3 \Gamma-4)}{(5 \Gamma-6)} \frac{M_{\mathrm{hot}}^{2}}{R_{\mathrm{hot}}}
$$

Conservation of rest-mass and total energy imply $M_{\text {hot }}=$ $2 M_{\text {cold }}$ and $E_{\text {hot }}=E_{\text {cold }}$, which, together with Eqs. (2) and (3), require $R_{\text {hot }}=2 R_{\text {cold }}$. The mass-radius relationship for a polytrope,

$$
M \propto R^{\frac{3-n}{1-n}} K^{\frac{n}{n-1}}
$$

may then be used to determine $K_{\text {hot }}$ :

$$
\frac{K_{\mathrm{hot}}}{K_{\mathrm{cold}}}=\left(\frac{M_{\mathrm{hot}}}{M_{\mathrm{cold}}}\right)^{\frac{n-1}{n}}\left(\frac{R_{\mathrm{hot}}}{R_{\mathrm{cold}}}\right)^{\frac{3-n}{n}}=4^{\frac{1}{n}} .
$$


We test the validity of Eq. (5) by comparing with the Newtonian simulations of RS, who monitor the timeevolution of the total entropy for a $\Gamma=2$ head-on collision (see Fig 7 of their paper). Once the remnant settles down, RS find that the entropy levels off at a value $S /\left(M_{\text {cold }} k_{\mathrm{B}} / \mu\right)=2 \ln \left(K_{\text {hot }} / K_{\text {cold }}\right) \approx 3$. Hence $K_{\text {hot }} / K_{\text {cold }} \approx 4.5$, which is in reasonable agreement with the analytic prediction of Eq. (5), $K_{\text {hot }} / K_{\text {cold }} \approx 4$.

\section{GENERAL RELATIVISTIC ANALYSIS}

Here we reconsider the same problem in the context of general relativity. We again assume that the two polytropic stars collide head-on from rest at infinity, conserving both their total rest mass, $2 M_{0}$ and total massenergy, $2 M$. At $t=0$ the two spherical stars are widely separated so that their mass-energies, like their restmasses, add linearly. We show that there exists a stable equilibrium solution for the remnant and determine the value of $K_{\text {hot }}$ corresponding to this solution.

Consider a sequence, parametrized by the central density, of spherical equilibrium stars constructed from the same one-parameter equation of state. The equilibrium models are solutions of the Oppenheimer-Volkoff (OV) equation of hydrostatic equilibrium. Those solutions which are stable against radial collapse reside on the ascending branch of the $M v s . \rho_{c}$ equilibrium curve, where $d M / d \rho_{c}>0$. Each of our colliding neutron stars is situated on the stable branch of the OV spherical equilibrium curve for a polytrope of constant $K=K_{\text {cold }}$ at the start of its infall. We want to determine where the remnant resides on the corresponding curve for a polytrope of constant $K=K_{\text {hot }}$ once the stars merge and settle down (see Fig 1).

The solutions exhibit a simple scaling behavior with respect to $K$ :

$$
\begin{array}{cl}
M\left(\rho_{c}\right)=K^{\frac{n}{2}} m^{*}\left(\rho_{c}^{*}\right), & M_{0}\left(\rho_{c}\right)=K^{\frac{n}{2}} m_{0}^{*}\left(\rho_{c}^{*}\right) \\
R\left(\rho_{c}\right)=K^{\frac{n}{2}} r^{*}\left(\rho_{c}^{*}\right), \quad \rho_{c}=K^{-n} \rho_{c}^{*} .
\end{array}
$$

In Eq. (6) $m^{*}, m_{0}^{*}$ and $r^{*}$ are universal, nondimensional functions of the nondimensional central density $\rho_{c}^{*}$ and depend only on the index $n$. The universal equilibrium curves for an $n=1$ polytrope are shown in Fig 2 .

The rest-mass and total mass-energy curves have simultaneous turning points at $\rho_{c}^{*}=\rho_{\max }^{*}$, at which point both masses take on their maximum values along the sequence. The ratio $m^{*} / m_{0}^{*}$ measures the specific binding energy of a star according to $E_{\text {bind }} / M_{0}=1-m^{*} / m_{0}^{*}$. This universal ratio monotonically decreases with $\rho_{c}^{*}$ along the stable branch of the OV curve, $\rho_{c}^{*} \leq \rho_{\max }^{*}$, as shown in Fig 3 . Conservation of rest mass and total mass-energy imply

$$
M_{0, \mathrm{hot}}=2 M_{0} \text { and } M_{\mathrm{hot}}=2 M \text {. }
$$

Combining Eqs. (6) and (7) gives

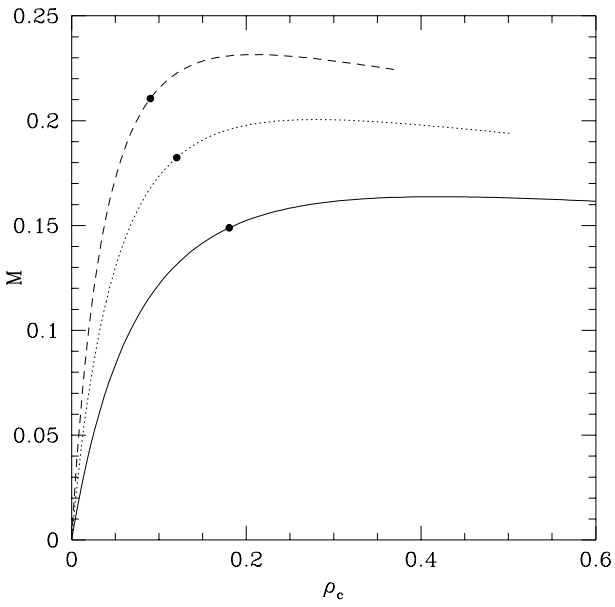

FIG. 1. Total mass-energy versus central density along the OV equilibrium curve for $n=1$ polytropes. The solid line shows models for $K=1$, the dotted line for $K=1.5$ and the dashed line for $K=2$. Solid points locate representative stable models with the same universal profile and binding energy.

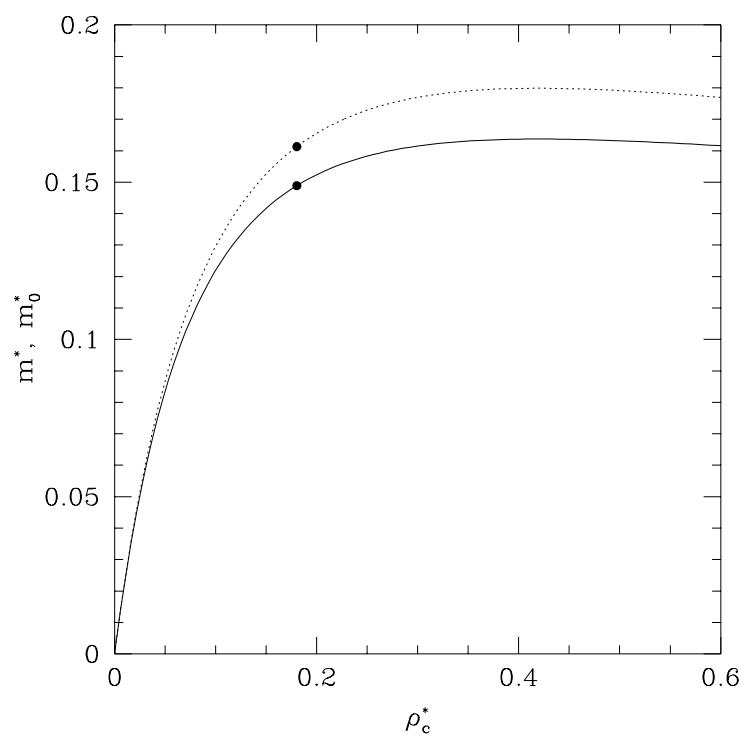

FIG. 2. Mass versus central density along the universal OV equilibrium curve for an $n=1$ polytrope. The solid line gives the total mass-energy, the dotted curve gives the rest mass. Solid points locate the representative stable model to which all the points in Fig 1 correspond. 


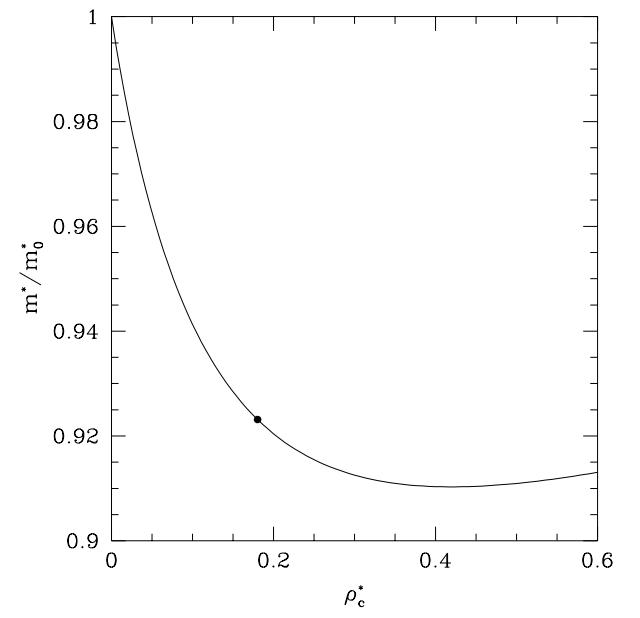

FIG. 3. Binding energy parameter versus central density along the universal OV equilibrium curve for an $n=1$ polytrope. The solid point locates the representative stable model to which all three points in Fig 1 correspond.

$$
m_{0, \text { hot }}^{*} / m_{0}^{*}=m_{\text {hot }}^{*} / m^{*}=2\left(K_{\text {hot }} / K_{\text {cold }}\right)^{-\frac{n}{2}},
$$

which implies

$$
m_{\mathrm{hot}}^{*} / m_{0, \mathrm{hot}}^{*}=m^{*} / m_{0}^{*} .
$$

The nondimensional binding energy ratio of the merged, hot remnant has the same value as in the cold progenitors. Because this ratio is a monotonic function of $\rho_{c}^{*}$, the remnant can relax to the same stable, equilibrium point on the universal binding energy curve, hence

$$
m_{0, \text { hot }}^{*}=m_{0}^{*} \text { and } \mathrm{m}_{\text {hot }}^{*}=\mathrm{m}^{*} .
$$

Thus, up to a scale factor $K$, the remnant profile is identical to that of its progenitors. Substituting Eq. (10) back into (8) implies

$$
\frac{K_{\text {hot }}}{K_{\text {cold }}}=4^{\frac{1}{n}},
$$

whereby we recover the result obtained in the Newtonian analysis (cf. Eq. (5).

\section{DISCUSSION AND CAVEATS}

Head-on collision and merger from rest at infinity need not lead to immediate collapse, regardless of the mass of the incident stars. Sufficient thermal pressure will always be generated to create a stable equilibrium configuration of higher entropy to which the fluid can can relax following merger: at fixed $\rho$

$$
P_{\text {therm }} \approx P-P_{\text {cold }} \approx P_{\text {cold }}\left(4^{\frac{1}{n}}-1\right),
$$

where $P_{\text {cold }}$ is given by Eq. (1) with $K=K_{\text {cold }}$ and where

$$
P_{\text {cold }} / \rho_{\text {cold }} \sim M / R \text {. }
$$

Setting the thermal pressure in the hot remnant equal to the pressure of a Maxwell-Boltzmann neutron gas yields

$$
k_{B} T \sim \frac{M m_{n}}{R} \frac{\rho_{\text {cold }}}{\rho_{\text {hot }}}\left(4^{\frac{1}{n}}-1\right) \sim 140 \mathrm{MeV}\left(\frac{M}{M_{\odot}}\right)\left(\frac{R}{10 \mathrm{~km}}\right)^{-1} .
$$

It is this thermal pressure that supports the remnant against collapse on a dynamical timescale $(\sim \mathrm{msec})$. The dominant cooling mechanism is thermal neutrino radiation, but the timescale is comparatively long $(\sim 10 \mathrm{sec})$ so the remnant evolves quasi-statically.

It is straightforward to modify our argument to account for the escape of baryons, the loss of energy due to gravitational radiation, the release of the progenitor stars from rest at finite separation rather than from infinity, etc. In the later two circumstances we simply replace Eq. (7) by

$$
M_{0, \text { hot }}=2 M_{0} \text { and } \mathrm{M}_{\mathrm{hot}}=2 \epsilon \mathrm{M},
$$

where $\epsilon \leq 1$ measures the decrease in the total massenergy below the initial value at infinite separation. Repeating the argument provides a constraint on $\epsilon$ for the remnant to relax to a dynamically stable quasiequilibrium state:

$$
\frac{m_{\mathrm{hot}}^{*}}{m_{0, \mathrm{hot}}^{*}}=\epsilon \frac{m^{*}}{m_{0}^{*}} \geq\left(\frac{m^{*}}{m_{0}^{*}}\right)_{\min } \Longrightarrow \epsilon \geq \frac{\left(\frac{m^{*}}{m_{0}^{*}}\right)_{\min }}{\left(\frac{m^{*}}{m_{0}^{*}}\right)},
$$

where $\left(m^{*} / m_{0}^{*}\right)_{\min }$ is the minimum value of the binding energy parameter along the stable branch. This minimum occurs at the maximum mass configuration and is $\approx 0.9$ for typical neutron stars (see Fig. 2). The low expected radiation efficiency $f=1-\epsilon \ll 1$ [1] [2] [5] implies that all head-on collisions from rest at infinity can lead to quasi-equilibrium except for those progenitors with masses within $f$ of the maximum. For collisions starting from rest at finite separation $d$, the total mass-energy is decreased by the initial binary interaction energy, so that

$$
M_{\mathrm{hot}} \approx 2 M-M^{2} / d, \quad(d \gg M) .
$$

Equating Eqs. (15) and (17) and ignoring radiation losses implies $\epsilon \approx 1-M /(2 d)$, whereby the condition for stable equilibrium becomes

$$
d \gtrsim \frac{M}{2}\left[1-\left(\frac{m^{*}}{m_{0}^{*}}\right)_{\min }\left(\frac{m^{*}}{m_{0}^{*}}\right)^{-1}\right]^{-1} .
$$

As $M \rightarrow M_{\max }$ and $\left(m^{*} / m_{0}^{*}\right) \rightarrow\left(m^{*} / m_{0}^{*}\right)_{\min }$, we require that $d \rightarrow \infty$ for stable equilibrium [6]. 
Simulations of head-on collisions of neutron stars in full general relativity have been performed [5] [7], but no systematic studies have been published. The reported cases exhibit dynamical behavior qualitatively similar to the Newtonian simulations regarding the recoil shocks, bounce and damped oscillations following the collision, and there is no collapse [8]. This result is consistent with our theoretical expectations, but we await a more complete numerical investigation for confirmation. There is the possibility that the configuration might greatly overshoot the final, allowed equilibrium state during the merger process and plunge immediately to a black hole, but such a fate is not evident. Hopefully, this paper may stimulate further numerical experiments, including examples which employ a more realistic hot, nuclear equation of state. Off-axis collisions are also of interest; while shock heating will be less important in this case, the angular momentum acquired by the remnant may supplement the reduced thermal pressure to prevent sudden collapse [9]. Resolving the fate of a head-on collision might serve as a proving ground for a relativistic code designed to handle these more complicated 3D binary scenarios.

\section{ACKNOWLEDGMENTS}

We thank A. Abrahams and T. Baumgarte for useful discussions. This work has been supported in part by NSF Grant AST 96-18524 and NASA Grant NAG5-7152.

[1] D.L. Gilden and S.L. Shapiro, Astrophys. J. 287, 728 (1984).

[2] F.A. Rasio and S.L. Shapiro, Astrophys. J. 401, 226 (1992).

[3] C.R. Evans in Proc. of 13th Texas Symposium on Relativistic Astrophysics, ed. M.P. Ulmer (Singapore: World Scientific), p. 152 (1987).

[4] The initial recoil shocks alone may generate nearly constant entropy when the equation of state is sufficiently stiff, in which case the progenitor stars are nearly homogeneous and the pre-shock gas parameters nearly uniform. Neutrino diffusion and convection may further reduce the entropy gradient.

[5] A.M. Abrahams and C.R. Evans in Proceedings of the 6th Marcel Grossman Meeting on General Relativity, ed F. Sato and T. Nakamura (Singapore: World Scientific), p. 1331 (1992).

[6] One must be especially careful to obey constraint (18) when setting up a numerical simulation, where it is not possible to start the stars off at infinite separation.

[7] L.L. Smarr and J.R. Wilson as reported by J. Wilson in Sources of Gravitational Radiation, ed. L.L. Smarr (Cambridge: Cambridge University Press), p. 423 (1979).
[8] The gravitational waveform for the relativistic simulation reported in Refs 5 matches well with the Newtonian result reported in Ref [2]; see Fig 8 of Ref [2]. The waveform probes the bulk motion of the coalescing matter and therefore attests to the similarity of the behavior.

[9] T.W. Baumgarte and S.L. Shapiro, Astrophys. J. in press (1998); astro-ph/9801294 